\title{
A Novel Role of the WNT-Dishevelled-GSK3 $\beta$ Signaling Cascade in the Mouse Nucleus Accumbens in a Social Defeat Model of Depression
}

\author{
Matthew B. Wilkinson, ${ }^{1}$ Caroline Dias, ${ }^{1}$ Jane Magida, ${ }^{1}$ Michelle Mazei-Robison, ${ }^{1}$ MaryKay Lobo, ${ }^{1}$ Pamela Kennedy, ${ }^{1}$ \\ David Dietz, ${ }^{1}$ Herbert Covington III, ${ }^{1,2}$ Scott Russo, ${ }^{1}$ Rachael Neve, ${ }^{3}$ Subroto Ghose, ${ }^{4}$ Carol Tamminga, ${ }^{4}$ \\ and Eric J. Nestler ${ }^{1}$ \\ ${ }^{1}$ Fishberg Department of Neuroscience and Friedman Brain Institute, Mount Sinai School of Medicine, New York, New York 10029, ${ }^{2}$ Department of \\ Psychology and Neuroscience, Duke University, Durham, North Carolina 22708, ${ }^{3}$ Massachusetts Institute of Technology, Cambridge, Massachusetts 02139, \\ and ${ }^{4}$ Department of Psychiatry, University of Texas Southwestern Medical Center, Dallas, Texas 75390
}

Based on earlier gene expression and chromatin array data, we identified the protein, dishevelled (DVL)-2, as being regulated in the nucleus accumbens (NAc), a key brain reward region, in the mouse social defeat model of depression. Here, we validate these findings by showing that DVL2 mRNA and protein levels are downregulated in NAc of mice susceptible to social defeat stress, effects not seen in resilient mice. Other DVL isoforms, DVL1 and DVL3, show similar patterns of regulation. Downregulation of DVL was also demonstrated in the NAc of depressed humans examined postmortem. Interestingly, several members of the WNT (Wingless)-DVL signaling cascade, including phospho-GSK3 $\beta$ (glycogen synthase kinase-3 $\beta$ ), also show significant downregulation in the NAc of susceptible, but not resilient, mice, demonstrating concerted regulation of this pathway in the NAc due to social defeat stress. By using viral-mediated gene transfer to overexpress a dominant-negative mutant of DVL in NAc, or by using a pharmacological inhibitor of DVL administered into this brain region, we show that blockade of DVL function renders mice more susceptible to social defeat stress and promotes depressionlike behavior in other assays. Similar prodepression-like effects were induced upon overexpressing GSK3 $\beta$ in the NAc, while overexpressing a dominant-negative mutant of GSK3 $\beta$ promoted resilience to social defeat stress. These findings are consistent with the knowledge that downregulation of DVL and phospho-GSK3 $\beta$ reflects an increase in GSK3 $\beta$ activity. These studies reveal a novel role for the DVL-GSK $3 \beta$ signaling pathway, acting within the brain's reward circuitry, in regulating susceptibility to chronic stress.

\section{Introduction}

Depression continues to exact enormous costs on society: one in six individuals in the U.S. will develop depressive symptoms requiring treatment, and depression is a leading cause of disability worldwide (Krishnan and Nestler, 2008). Available antidepressants, all of which target the brain's monoamine systems, can be highly effective in some patients, but more than half of all depressed individuals remain symptomatic despite optimal therapy. A major goal of current research is to better understand the neurobiology of depression so that more effective treatments can be developed.

We have used chronic social defeat stress in mice as a useful animal model of depression and other stress-related disorders.

Received Jan. 3, 2011; revised April 26, 2011; accepted May 5, 2011.

Author contributions: M.B.W., C.D., S.R., and E.J.N. designed research; M.B.W., J.M., M.M.R., M.K.L., P.K., D.D., H.C., S.R., R.N., and S.G. performed research; M.B.W., S.R., S.G., and C.T. contributed unpublished reagents/analytic tools; M.B.W., S.R., and C.T. analyzed data; M.B.W., C.T., and E.J.N. wrote the paper.

We thank Mariann Bienz for the DVL2 mutant vectors, Richard Jope for the GSK3 $\beta$ vectors, and Patricia Salinas for the DVL1 vector.

The authors declare no competing financial interests.

Correspondence should be addressed to Eric J. Nestler, Fishberg Department of Neuroscience and Friedman Brain Institute, Mount Sinai School of Medicine, One Gustave L. Levy Place, New York, NY 10029. E-mail: eric.nestler@mssm.edu.

DOI:10.1523/JNEUROSCI.0039-11.2011

Copyright $\odot 2011$ the authors $\quad 0270-6474 / 11 / 319084-09 \$ 15.00 / 0$
Chronic defeat stress induces a range of depression-like symptoms, including a profound social avoidance, which can be reversed by chronic, but not acute, administration of standard antidepressant medications (Berton et al., 2006; Tsankova et al., 2006). Moreover, not all mice subjected to such repeated stress develop these symptoms, which has allowed the use of the social defeat paradigm to study the neurobiology of resilience as well (Krishnan et al., 2007). While numerous brain regions likely contribute to susceptibility and resilience to social defeat stress, our work has focused on a crucial role of the nucleus accumbens (NAc) and related regions of the brain's reward circuitry in determining susceptibility versus resilience to social defeat stress. Genome-wide analyses of gene expression and chromatin changes in the NAc of susceptible and resilient mice have revealed possible molecular mechanisms underlying responses to chronic social defeat stress (Berton et al., 2006; Krishnan et al., 2007; Covington et al., 2009; Wilkinson et al., 2009).

For example, in a recent genome-wide analysis of repressive histone methylation in NAc of mice subjected to chronic social defeat stress, we identified numerous genes where stress induced changes in histone methylation in susceptible but not resilient mice (Wilkinson et al., 2009). Prominent among these genes was dishevelled (DVL)-2, a key step in the Wingless (WNT)-Frizzled 
signaling cascade (Gao and Chen, 2010), which has been extensively characterized in cancer and developmental biology, and more recently has been investigated in several psychiatric models (see Discussion, below). Under basal conditions, DVL is maintained in the cytoplasm in an inactive, depolymerized form. WNT, secreted from afferent cells, activates Frizzled, a plasma membrane receptor, which then triggers the binding and polymerization of DVL. The primary downstream signaling mechanism of DVL is termed the canonical pathway, where DVL activation leads to its binding of Axin, phosphorylation and inhibition of glycogen synthase kinase- $3 \beta$ (GSK $3 \beta$ ), and the regulation of several downstream targets, including $\beta$-catenin (Gao and Chen, 2010).

In the present study, we focused on regulation of the WNT-DVLGSK $3 \beta$ signaling pathway in NAc by chronic social defeat stress. We demonstrate concerted regulation of multiple proteins in this pathway, including all three isoforms of DVL (DVL1-3) and GSK3 $\beta$, in susceptible but not resilient mice, and provide direct, causal evidence that such regulation represents a prodepression-like maladaptation that promotes susceptibility to chronic stress.

\section{Materials and Methods}

Social defeat stress paradigm and social interaction test. Male C57 mice (7-9 weeks old) were obtained from Jackson Laboratory and housed on a $12 \mathrm{~h}$ light-dark cycle with access to food and water ad libitum. Male CD1 retired breeder mice (9-13 months old) were obtained from Charles River Laboratories. All animal procedures were approved by Mount Sinai's Institutional Animal Care and Use Committee. The total number of animals used in these experiments was 240 .

Mice, acclimated to the facility for 1 week before any experimentation, were subjected to chronic social defeat stress exactly as described previously (Berton et al., 2006; Krishnan et al., 2007). CD1 mice, prescreened for a threshold level of aggressive behavior, were used to defeat the C57 mice. The $\mathrm{C} 57$ mice were defeated for $10 \mathrm{~min}$ per day and then housed for the remainder of the day with the aggressor but separated by a Plexiglas screen. This process was repeated daily for 10 consecutive days with a different CD1 aggressor each day. Control animals were housed in divided cages with another C57 mouse. On day 11, defeated animals were subjected to a social interaction test and sorted into either susceptible or unsusceptible (resilient) phenotypes based on interaction scores (Krishnan et al., 2007).

Briefly, during the social interaction test, the mice were placed in an open field $(42 \times 42 \mathrm{~cm})$ with a small empty Plexiglas cage placed on one wall. The amount of time the animal spent in the area around this cage was measured for $2.5 \mathrm{~min}$. A novel CD1 mouse was then introduced into the Plexiglas cage and the procedure was repeated. The tests were performed under red light. Those animals that spent more time in the interaction zone when a novel mouse target was present compared with when it was absent were considered resilient (Krishnan et al., 2007). Those animals that spent less time in the interaction zone when the target was present compared with when it was absent were considered susceptible. In general, control animals spent more time in the interaction zone when the target was present. Animals were killed $1 \mathrm{~d}$ later for biochemical analyses.

For the submaximal defeat paradigm (see Figs. 4, 5), C57 mice were subjected to repeated defeat episodes over $1 \mathrm{~d}$. Animals were placed into the $\mathrm{CD} 1$ aggressor's cage for $5 \mathrm{~min}$, followed by a $10 \mathrm{~min}$ break. This was repeated two further times with different CD1 aggressors. This submaximal defeat paradigm has been used by our group previously to test whether an experimental manipulation (e.g., viral gene transfer or drug treatment) might potentiate an animal's susceptibility to defeat stress (Krishnan et al., 2007). Such submaximal $1 \mathrm{~d}$ defeats were done after any other behavioral testing (sucrose preference, forced swim test).

In other cases, i.e., for the dominant-negative GSK3 $\beta$ ( $\operatorname{dnGSK} 3 \beta)$ data (see Fig. 5) and studies of wild-type DVL, a full course of social defeat was performed on an accelerated schedule (twice a day for $10 \mathrm{~min}$ per day for $4 \mathrm{~d} ; 8$ total defeats). We have shown that this accelerated paradigm, which
Table 1. Gene primers used for DVL pathway analysis

\begin{tabular}{|c|c|c|}
\hline Primer & Forward sequence & Reverse sequence \\
\hline DVL 1 & AGTGGAGCCTCAGATCAGGA & GGTCCTGGGTACTGGTAGGG \\
\hline DVL2 & TGACAATGACGGTTCCAGTG & GCGCTGGATACTGGTAGGAG \\
\hline DVL 3 & CTACACGCAGCAGTCTGAGG & CATAGCTTGGGTGTGTGTGG \\
\hline$\alpha$-Catenin & TCCCAGGCATGTCTACAACA & GTAGCCTGGGCTGCATTAGA \\
\hline$\beta$-Catenin & TATTGACGGGCAGTATGCAA & СCCTCATCTAGCGTCTCAGG \\
\hline GSK3 $\beta$ & ACAGGCCACAGGAAGTCAGT & GATGGCAACCAGTTCTCCAG \\
\hline Akt & CTGGAGGACAACGACTACGG & CATGAGGATCAGCTCGAACA \\
\hline Groucho & CAAATGCAGCCTGTTCCTTT & GTCACTGCACACACCACCTC \\
\hline Wnt 2a & GGCGACTATCTCTGGAGGAA & ACGAGGTCATTTTTCGTTGG \\
\hline Wnt 5a & AGGAGTTCGTGGACGCTAGA & CTACGGCCTGCTTCATTGTT \\
\hline Wnt 5b & AGCCGTACTCCACGTTGTCT & AGAGAGGGTGAGCTGTCCAC \\
\hline Prop1 & CCTAAGCTTTGCCCACAGAG & AAGGCTGACTCCAGCTGTTC \\
\hline Axin & CCACCACCATGTTCACCATA & TGGCATGACCATGTGTTTCT \\
\hline Chibby & GTGATTAGTGGGGGTGTGGA & CCGCAGGAGATTGTTTTCTT \\
\hline Naked & TACCAGCTTGCTGCATACCA & СTTACTCTGGCTCCCGTCAG \\
\hline Frizzled 1 & CTTTCTGGGGGAGAAGGACT & ACCAGATGCCTATCCAGGTG \\
\hline Frizzled 6 & GTGCTGCAAGAGTCCTGTGA & GTCGCTCCTGTGCTAGTTCC \\
\hline
\end{tabular}

is sufficient to induce maximal avoidance behavior in control animals, is necessary due to the transient transgene expression of Herpes simplex virus (HSV) vectors (Krishnan et al., 2007).

$R N A$ isolation and PCR analysis of WNT- $\beta$-catenin signaling pathway. NAc samples were collected and processed as described previously (Covington et al., 2009). Briefly, bilateral 14 gauge tissue punches were taken on ice from animals following rapid decapitation. Samples were placed on dry ice and stored at $-80^{\circ} \mathrm{C}$. For RNA isolation, samples were thawed on ice. TriZol (Invitrogen) was added and samples were homogenized. Chloroform was added and samples were spun to separate layers. The clear layer was then processed with an RNAeasy MicroKit (Qiagen). Samples were analyzed with NanoDrop and 500 ng of RNA was reverse transcribed using a qScript kit (VWR). The cDNA was diluted to $500 \mu \mathrm{l}$ and $3 \mu \mathrm{l}$ were used per quantitative PCR. The reaction mixture consisted of Perfecta SYBR Green $(5 \mu \mathrm{l})$, forward and reverse primers $(0.5 \mu \mathrm{l}$ each), water $(1 \mu \mathrm{l})$, and $\mathrm{cDNA}$ template. Samples were then heated to $95^{\circ} \mathrm{C}$ for $2 \mathrm{~min}$ followed by 40 cycles of $95^{\circ} \mathrm{C}$ for $15 \mathrm{~s}, 60^{\circ} \mathrm{C}$ for $33 \mathrm{~s}$, and $72^{\circ} \mathrm{C}$ for 33 s. Analysis was done via the $\Delta \Delta \mathrm{C}(\mathrm{t})$ method (Tsankova et al., 2006). Samples were normalized to GAPDH. Primer pairs are shown in Table 1.

Viral vector construction. Wild-type and dominant-negative (K85A, K86A) GSK3 $\beta$ cDNAs were provided by Dr. Richard Jope (University of Alabama-Birmingham, Birmingham, AL). PCR primers were generated to the $3^{\prime}$ and $5^{\prime}$ end of the GSK3 $\beta$ cDNAs to add KpnI and EcoRI restriction sites, respectively. PCR products were then digested, purified, and ligated into the KpnI and EcoRI sites in the HSV p1005 plasmid, which also expressed GFP under a separate transcriptional unit (Clark et al., 2002). Correct p1005 ligation was verified by sequencing.

Wild-type DVL1 and a DVL1 construct containing a deletion of the PDZ domain (DVL1- $\triangle \mathrm{PDZ}$ ) of the protein were obtained from Dr. Patricia Salinas (University College London, London, UK). DVL2 was obtained from Dr. Mariann Bienz (University of Cambridge, Cambridge, UK). DVL1 and DVL1- $\triangle$ PDZ were cut with EcoRI $\left(5^{\prime}\right)$ and XhoI $\left(3^{\prime}\right)$ and cloned into p1005-HSV plasmid cut with the same enzymes. DVL2, DVL2-M2, and DVL2-M4 were cut with BamHI (5') and PmeI ( $\left.3^{\prime}\right)$ and cloned into BamHI (5') and EcoRV (3') cut p1005-HSV plasmid. All HSV vectors were packaged, titered, and validated as before (Neve et al., 2005).

Intra-NAc infusion of viral constructs and minipump placement. HSV surgeries were performed as previously described (Krishnan et al., 2007). Briefly, animals were anesthetized with ketamine $(100 \mathrm{mg} / \mathrm{kg})$ and xylazine $(10 \mathrm{mg} / \mathrm{kg}$ ) and placed into a stereotaxic apparatus (Kopf Instruments). The skin was incised along the midline and bregma located. Burr holes were drilled, a Hamilton syringe needle was lowered, and the vectors were infused bilaterally at a rate of $0.1 \mu \mathrm{l}$ per minutes to a total volume of $0.5 \mu$ l. Coordinates for the needle tip as measured from bregma were as follows: anteroposterior, +1.6 ; lateral, \pm 1.5 ; dorsoventral, $-4.4 \mathrm{~mm}$. The needle remained in place for $5 \mathrm{~min}$ following the 
injection to limit suction of the vector up the needle track. Skin incisions were glued together using VetBond $(3 \mathrm{M})$ and animals recovered overnight before any behavior was performed. Mice were used 1-2 d after HSV surgery given the relatively short expression time of the viral vectors, which makes longer recovery times impossible. We have noted in numerous prior studies that the animals are fully recovered at this time point and show no untoward effects (Krishnan et al., 2007; Vialou et al., 2010).

Osmotic minipump surgery was performed as previously described (Covington et al., 2009). Animals were anesthetized with a combination of ketamine $(100 \mathrm{mg} / \mathrm{kg})$ and xylazine $(10 \mathrm{mg} / \mathrm{kg})$ and surgically implanted with two subcutaneous Alzet minipumps (model 1002; Durect) and guide cannulae (Plastics One) targeting the NAc bilaterally. One day before surgery, two cannulae (28 gauge stainless steel) were filled with sulindac ( $1.5 \mathrm{~mm}$; Sigma) or 5\% hydroxypropyl $\beta$-cyclodextrin vehicle (Trappsol; CTD), and each pedestal within the assembly was separately affixed via vinyl tubing to a minipump, each loaded with drug or vehicle. The minipumps were activated on the evening before surgery (by incubating them at $40^{\circ} \mathrm{C}$ ) to initiate a continuous delivery at $0.25 \mu \mathrm{l} / \mathrm{h}$ over $14 \mathrm{~d}$. Cannulae, targeting the NAc according to bregma (anteroposterior, +1.5 ; mediolateral, \pm 1.0 ; dorsoventral, -4.5 ) were permanently fixed to the skull with Loctite skull adhesive (Henkel). The coordinates used for minipump surgeries were slightly different from those used for viral vector infusions to account for the hardware that is attached to the skull in the former. Cannulae, tubing, and minipumps were all secured under the skin using Vetbond tissue adhesive (3M) and two staples. Mice recovered from surgery for $2 \mathrm{~d}$ before beginning behavioral tests to match the recovery times used in the HSV experiments.

Sucrose preference and forced swim tests. Sucrose preference was performed following previously established protocols (Covington et al., 2009). Animals were given two identical $50 \mathrm{ml}$ bottles with sipper tubes (Ancare) containing water for $2 \mathrm{~d}$. The third day, animals were provided with one bottle containing $1 \%$ sucrose and one bottle containing water. The amount of sucrose and water consumed was measured each day for the next $4 \mathrm{~d}$ and reported as percentage of sucrose consumed (sucrose volume/total volume $\times 100$ )

The forced swim test was performed as described previously (Krishnan et al., 2007). Animals were placed in a $4 \mathrm{~L}$ beaker containing $3 \mathrm{~L}$ of water at a temperature of $25 \pm 1^{\circ} \mathrm{C}$. Tracking was performed with Ethovision for a period of $6 \mathrm{~min}$. The experiment was performed under white light. Animals were analyzed for the amount of time spent mobile versus immobile.

The same mice within each treatment group were used for all behavioral experiments. The overall time course of the experiments was such that animals were first analyzed for sucrose preference on days 1-4 postsurgery, and then for forced swimming on days 2 or 3 (virus or minipump, respectively). Social defeat studies were initiated on day 3 or 4 (virus or minipump, respectively) with social interaction measured $24 \mathrm{~h}$ after the last defeat. The short time course was dictated by the duration of viral expression or minipump delivery. Prior data have shown that this order of behavioral testing does not influence findings in the later tests (Krishnan et al., 2007).

Immunohistochemistry and Western blotting. Mice were sedated with chloral hydrate and perfused with $1 \times$ PBS followed by $4 \%$ paraformaldehyde in $1 \times \mathrm{PBS}$ at $\mathrm{pH}$ 7.4. Whole brains were postfixed in $4 \%$ paraformaldehyde overnight and subsequently cryoprotected in $30 \%$ sucrose in PBS. Brains were sliced as $35 \mu \mathrm{m}$ sections on a microtome and sections were stored in $1 \times$ PBS with sodium azide. Before use, the slices were washed in PBS. They were then blocked with 3\% donkey serum blocking buffer and incubated with 1:5000 chicken anti-GFP primary antibody (Aves Labs) overnight. Secondary donkey anti-chicken Cy2 antibody at 1:500 was followed with three washes of PBS. All sections were mounted and allowed to dry on Superfrost Plus slides (VWR). The specimens were dehydrated in ethanol and cleared in Citrisolv (Fisher), and finally coverslipped with DePex. Images were taken with an Axiophot fluorescent microscope (Zeiss).

Bilateral NAc punches, taken as described above, were sonicated in a detergent-based lysis buffer containing phosphatase and protease inhibitors. Samples were then centrifuged for $30 \mathrm{~min}$ at $14,000 \mathrm{RPM}$ at $4^{\circ} \mathrm{C}$ and
Table 2. Human postmortem tissue comparisons

\begin{tabular}{lllll}
\hline Diagnosis & Age & PMI & RIN & pH average \\
\hline Control & $39.7 \pm 6.8$ & $23 \pm 1.5$ & $8.6 \pm 0.3$ & $6.8 \pm 0.1$ \\
Depressed & $34.2 \pm 6.3$ & $19.1 \pm 1.2$ & $8.4 \pm 0.4$ & $6.8 \pm 0.1$ \\
$t$ Test & NS & NS & NS & NS \\
\hline
\end{tabular}

Age denotes the age of the subjects at their time of death. PMI (postmortem interval) indicates the time between death and preservation of brain tissue. RIN provides a measure of brain tissue quality. $\mathrm{pH}$ average is the $\mathrm{pH}$ of the brain tissue, another indicator of tissue quality. NS, Not significant. Data are presented as means \pm SEM.

the supernatant was collected and quantified with the $\mathrm{D}_{\mathrm{C}}$ kit (Bio-Rad). Laemelli sample buffer was added and equal amounts of protein were analyzed on $4-15 \%$ Tris-HCl Ready Gels (Bio-Rad); resulting gels were transferred to polyvinylidene difluoride membranes, which were blocked in $5 \%$ milk and incubated overnight with primary antibodies in 5\% BSA or milk. Primary antibodies were used as follows: DVL2, 1:500; $\beta$-catenin, 1:1000; GSK3- $\beta$, 1:5000; phospho-GSK3- $\beta$ (phospho Ser9), 1:500 (Cell Signaling Technology). Blots were then washed and incubated in peroxidaselabeled secondary antibody (Vector Labs). All samples were normalized to GAPDH (1:50,000; Cell Signaling Technology).

Human postmortem tissue. mRNA and protein from human NAc was analyzed as described previously (Covington et al., 2010; Vialou et al., 2010a,b); demographic data for the individuals analyzed here are included in these earlier publications. Human postmortem brain tissue was obtained from the Dallas Brain Collection, where tissue is collected from the Dallas Medical Examiner's Office and University of Texas (UT) Southwestern's Tissue Transplant Program following consent of next-ofkin. Tissue was analyzed from both males matched for age, postmortem interval, RNA integrity number (RIN), and $\mathrm{pH}$. Cases were subjected to a standard dissection before snap freezing in $-40^{\circ} \mathrm{C}$ isopentane and storage at $-80^{\circ} \mathrm{C}$; further dissection of NAc was performed on frozen tissue. The UT Southwestern Institutional Review Board reviewed and approved the collection of this tissue for research use. The total number of samples tested was 16 (eight documented depressed individuals and eight matched controls). There was no difference in age, postmortem interval, RNA integrity (as measured by RIN), or brain pH between the groups. All samples were from Caucasian males (Table 2).

Molecular pathway analysis. Lists of genes whose mRNA levels or chromatin state were found to be regulated in NAc by chronic social defeat stress or antidepressant treatments, derived from previous studies (Berton et al., 2006; Krishnan et al., 2007; Covington et al., 2009; Wilkinson et al., 2009) were used. Datasets of regulated genes were processed by Ingenuity pathway analysis (Ingenuity Systems) to identify highly regulated molecular signaling pathways.

Statistical analysis. All data are expressed as the mean \pm SEM. Mean differences between groups were determined using a Student's $t$ test or a one-way ANOVA, followed by Tukey post hoc tests when the main effect or interaction was significant at $p<0.05$. Statistical analyses were performed using Prism 5.0 (GraphPad Software).

\section{Results}

\section{Chronic social defeat stress regulates the DVL pathway} in NAc

Several previous studies from our laboratory applied genomewide methods to identify genes whose mRNA expression level or chromatin state is altered in the NAc by chronic social defeat stress or chronic antidepressant treatment (Berton et al., 2006; Krishnan et al., 2007; Covington et al., 2009; Wilkinson et al., 2009). Each of these datasets, which focused on regulation in susceptible versus resilient mice or the effects of different types of antidepressant treatments, was subjected to Ingenuity molecular pathway analysis. As illustrated in Figure 1, one of the most highly and consistently regulated pathways across all of these datasets was the WNT-DVL-GSK3 $\beta$ pathway. Prominent among the regulated genes in this pathway was DVL2, which displayed increased repressive histone methylation at its gene promoter in the NAc after chronic social defeat stress, with such regulation seen 


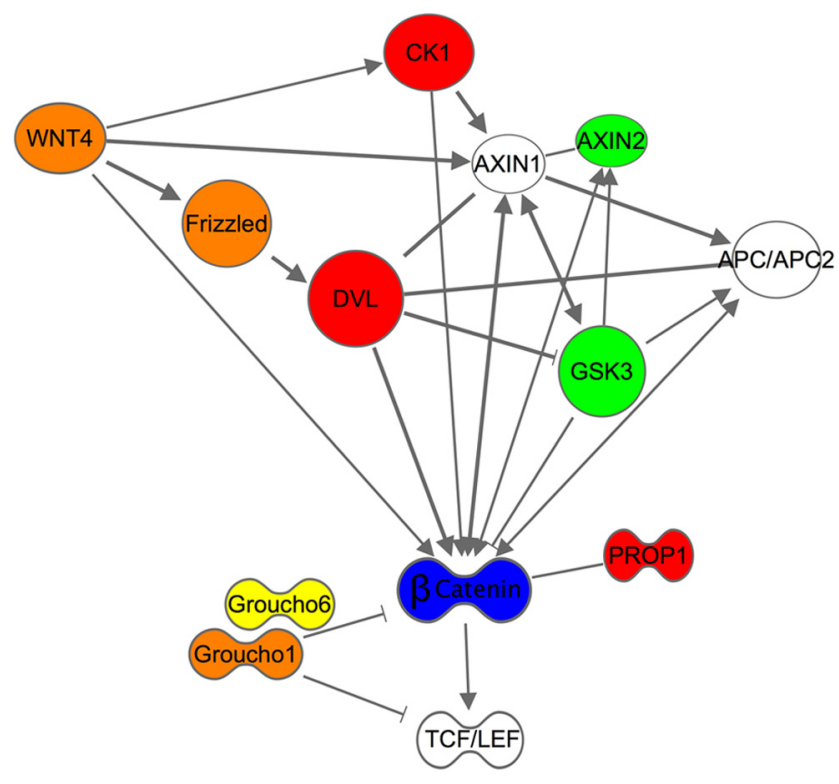

Figure 1. Concerted regulation of the WNT-DVL-GSK3 $\beta$ signaling pathway in NAc by chronic social defeat stress. Genes are color-coded according to regulation seen in this brain region in previous genome-wide studies of mRNA expression levels or altered repressive histone methylation (Berton et al., 2006; Krishnan etal., 2007; Covington et al., 2009; Wilkinson et al., 2009). Red, Genes regulated by chronic social defeat stress in susceptible mice with reversal seen in resilient mice and after chronic antidepressant treatment; green, genes regulated by chronic social defeat stress in susceptible mice with reversal seen with antidepressant treatment; blue, genes regulated by resilience only; orange, genes regulated in susceptible mice only; yellow, genes regulated by chronic antidepressant treatment only. Frizzled, DVL, CK1, PROP1, and GSK3 displayed evidence of downregulation in the NAc of susceptible mice; WNT4, Axin2, and Grouch01, upregulation in susceptibility; $\beta$-catenin, upregulation in resilience; and Groucho6, upregulation after antidepressant treatment. The statistical criteria for such regulation are provided in the text. CK1, casein kinase 1; APC, adenomatous polyposis coli; PROP1, PROP paired-like homeobox 1; TCF/LEF, T-cell factor/lymphoid enhancer factor; Groucho, also known as TLE (transducin-like enhancers of split).

only in susceptible mice, not in resilient mice, and reversed by chronic antidepressant treatment (Wilkinson et al., 2009).

Therefore, as a first step in validating these findings, we measured DVL2 mRNA levels in NAc on day $12(2 \mathrm{~d}$ after the last defeat episode and $1 \mathrm{~d}$ after the social interaction test) and found, consistent with our earlier data showing induction of repressive histone methylation at the $d v l 2$ gene promoter, that DVL2 expression is indeed decreased by $\sim 40 \%$ in the NAc of susceptible mice (one-way ANOVA: $F_{(2,26)}=14.70, p<0.0001$ ), with no significant change observed in resilient animals (Fig. 2A) compared with control animals (Tukey post hoc test). These susceptible and resilient subgroups were identified on the basis of social avoidance evident in susceptible animals, but not resilient animals (Fig. $2 B$ ). Susceptible animals were significantly more likely to avoid the target during the social interaction test compared with control or resilient animals (one-way ANOVA: $F_{(2,26)}=$ $11.28, p=0.0003)$. There was no statistical difference between the control and resilient animals, as evidenced by the Tukey post hoc test. The reduction in DVL2 mRNA expression seen in the NAc of susceptible mice was associated with an equivalent reduction in DVL2 protein (one-way ANOVA: $F_{(2,23)}=11.77, p=$ 0.0003 ) (Fig. 2C), with no difference seen between the control and resilient animals (Tukey post hoc test). As there are three known mammalian isoforms of DVL, we next analyzed mRNA levels of DVL1 and DLV3. We found that these other DVL family members exhibited similar patterns of regulation as observed for DVL2: significant reductions in the NAc of susceptible animals but not resilient animals. In fact, DVL1 showed significantly in-
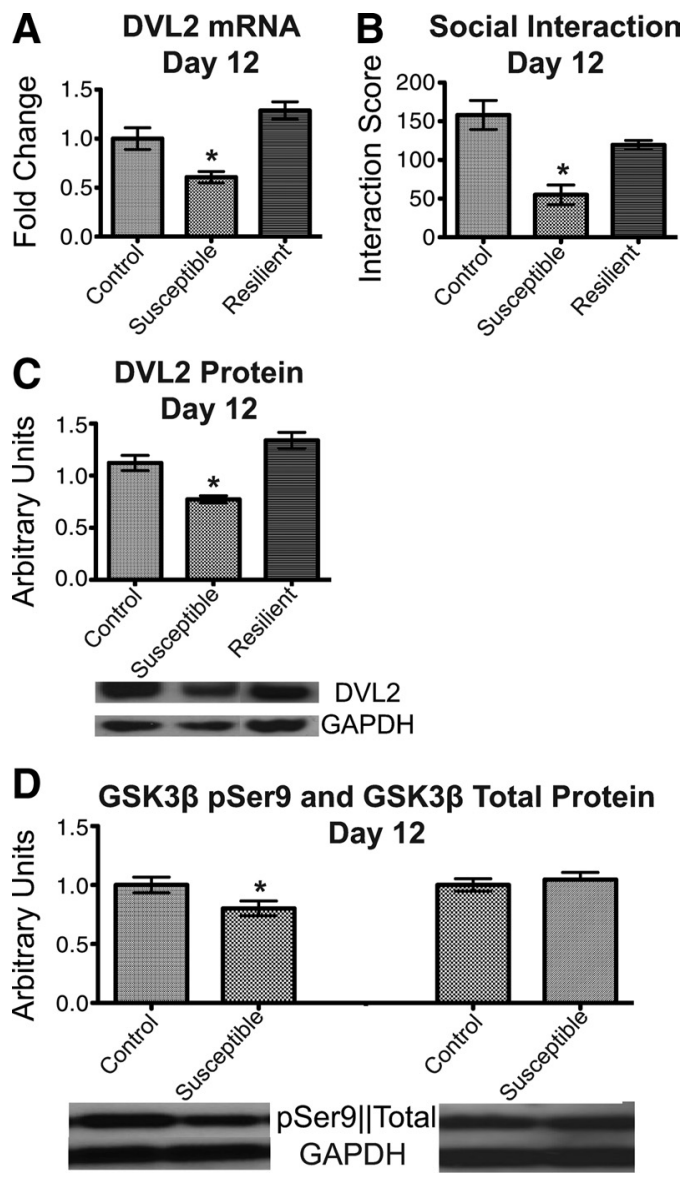

Figure 2. Regulation of DVL-GSK3 $\beta$ signaling in the NAc by chronic social defeat stress. $A$, DVL2 mRNA levels are significantly downregulated at day 12 in susceptible animals, whereas resilient animals show levels comparable to controls ( $n=8-10$ per group). $\boldsymbol{B}$, Social interaction data show increased avoidance in susceptible but not resilient animals $(n=8-10$ per group). C, Protein levels of DVL2 are significantly decreased in susceptible, but not resilient, animals compared with controls at day 12 ( $n=8-10$ per group). $\boldsymbol{D}$, Protein levels of an inactive form of GSK3 $\beta$ (pSer9) are significantly decreased at day 12 , while overall GSK3 $\beta$ protein levels are unchanged ( $n=6-8$ per group). Error bars are SEM $\left({ }^{*} p<0.05\right)$.

creased expression in the resilient subgroup (Table 3). Unfortunately, it was not possible to assay for levels of DVL1 or DVL3 protein in NAc due to the lack of suitable antibodies.

Given this robust regulation of DVL family proteins, we tested the effect of chronic social defeat stress on numerous proteins that are either upstream (e.g., WNT and Frizzled) or downstream (e.g., GSK3 $\beta$ ) in the DVL signaling pathway. As shown in Table 3, consistent with our molecular pathway analysis, numerous members of this signaling pathway, in addition to DVLs, including specific WNT subtypes, Axin, and GSK3 $\beta$, are significantly downregulated in the NAc of susceptible animals after chronic social defeat stress, with none of the changes observed in resilient animals. Together, these data reveal a profound and concerted regulation of DVL signaling in the NAc of susceptible mice. To directly assess the effect of social defeat stress on the functional activity of this pathway in the NAc, we analyzed total levels of GSK3 $\beta$ (Fig. 2D) as well as its phosphorylated form, phosphoSer9, which is functionally inactive (Fig. $2 D$ ). While total levels of GSK3 $\beta$ protein are unchanged (Student's $t$ test, $p=0.6, \mathrm{df}=14$ ), levels of its phosphorylated form are significantly reduced in the NAc of susceptible mice (Student's $t$ test, $p=0.047$, $\mathrm{df}=15$ ). This reduction in phospho-GSK3 $\beta$, which is indicative of an increase in GSK3 $\beta$ catalytic activity, is consistent with the down- 
Table 3. Fold changes for DVL pathway genes

\begin{tabular}{lllll}
\hline Gene & Control & Susceptible & Resilient & Statistics \\
\hline DVL1 & 1.00 & $0.62^{*}$ & $1.54^{*}$ & One-way ANOVA: $F_{(2,26)}=18.88, p<0.0001$ \\
DVL2 & 1.00 & $0.61^{*}$ & 1.29 & One-way ANOVA: $F_{(2,26)}=14.70, p<0.0001$ \\
DVL3 & 1.00 & $0.67^{*}$ & 1.02 & One-way ANOVA: $F_{(2,26)}=4.75, p=0.0178$ \\
$\alpha$-Catenin & 1.00 & $0.72^{*}$ & 1.32 & One-way ANOVA: $F_{(2,26)}=8.90, p=0.0011$ \\
GSK3 $\beta$ & 1.00 & $0.66^{*}$ & 1.17 & One-way ANOVA: $F_{(2,26)}=13.84, p<0.0001$ \\
Akt & 1.00 & $0.74^{*}$ & & Student's $t$ test: $p=0.006$ \\
Grouch0 & 1.00 & 0.65 & & Student's test: $p=0.079$ \\
Wnt 2a & 1.00 & $0.64^{*}$ & & Student's t test: $p=0.015$ \\
Wnt 5a & 1.00 & 0.75 & & Student's test: $p=0.096$ \\
Wnt 5b & 1.00 & 0.97 & & Student's test: $p=0.878$ \\
Pak1 & 1.00 & 0.93 & & Student's $t$ test: $p=0.479$ \\
Prop1 & 1.00 & 1.03 & & Student's test: $p=0.894$ \\
Axin & 1.00 & $0.74^{*}$ & & Student's $t$ test: $p=0.030$ \\
Chibby & 1.00 & 0.87 & & Student's test: $p=0.168$ \\
Naked & 1.00 & 0.63 & & Student's $t$ test: $p=0.084$ \\
Frizzled 6 & 1.00 & 0.76 & & Student's test: $p=0.064$ \\
Frizzled 1 & 1.00 & $0.60^{*}$ & & Student's $t$ test: $p=0.007$
\end{tabular}

Quantitative PCR data are presented as one-way ANOVAs or $t$ test, as appropriate. For DVL1, post hoc analyses show a significant increase in mRNA levels for resilient animals as compared to control. In all other cases, the resilient and control animals are not significantly different for the genes analyzed. Different from control ( $\left.{ }^{*} p<0.05\right)$.

regulation of DVL signaling observed in the NAc of susceptible mice. We also found that levels of both total and phosphoGSK $3 \beta$ are unchanged in the resilient animals compared with the control state (data not shown).

As our group has previously validated several biochemical changes observed in the social defeat paradigm in human depression (Krishnan et al., 2007, 2008; Covington et al., 2010; Vialou et al., 2010a,b), we investigated mRNA levels of all three DVL isoforms in the NAc of depressed humans examined postmortem. We found that mRNA levels of all three DVL isoforms were significantly decreased in depressed patients: DVL1 mRNA was decreased by $55 \%(p=0.042)$, DVL2 mRNA by $42 \%(p=0.031)$, and DVL3 mRNA by 39\% ( $p=0.041$; all analyses by one-tailed Student's $t$ tests, df $=14, n=8$ per group). We did not find changes at the mRNA level in any of the other genes listed in Table 1 (data not shown). This difference between our mouse social defeat model and human depression might reflect greater heterogeneity in the human samples, as would be expected. We also analyzed DVL2 protein and found that it was significantly decreased in the NAc of depressed patients, consistent with the human mRNA data and our findings in mouse social defeat (twotailed $t$ test, $p=0.013$, $\mathrm{df}=14, n=8$ per group). In contrast, several control proteins (e.g., GAPDH, $\beta$-actin) showed no difference between depressed and control humans, demonstrating specificity of our findings for DVL2.

\section{Downregulation of the DVL pathway in NAc promotes depression-like behavior}

We next studied the behavioral consequences of the downregulation of DVL signaling in the NAc of susceptible mice by constructing HSV vectors that overexpress wild-type forms of DVL or a dominant-negative mutant. The latter, DVL1- $\triangle \mathrm{PDZ}$, lacks a PDZ domain in the protein, which has been shown to be required for DVL polymerization and downstream signaling to GSK3 $\beta$ and $\beta$-catenin in several systems (Krylova et al., 2000; Uematsu et al., 2003; Grandy et al., 2009; Gao and Chen, 2010). We used DVL1 constructs, as opposed to DVL2 constructs, because the latter were not readily available. These HSV vectors were then injected bilaterally into the NAc of C57 mice and the mice were analyzed in a battery of behavioral tests. The discrete targeting of the NAc enabled by these vectors is illustrated in Figure $3 A$. This
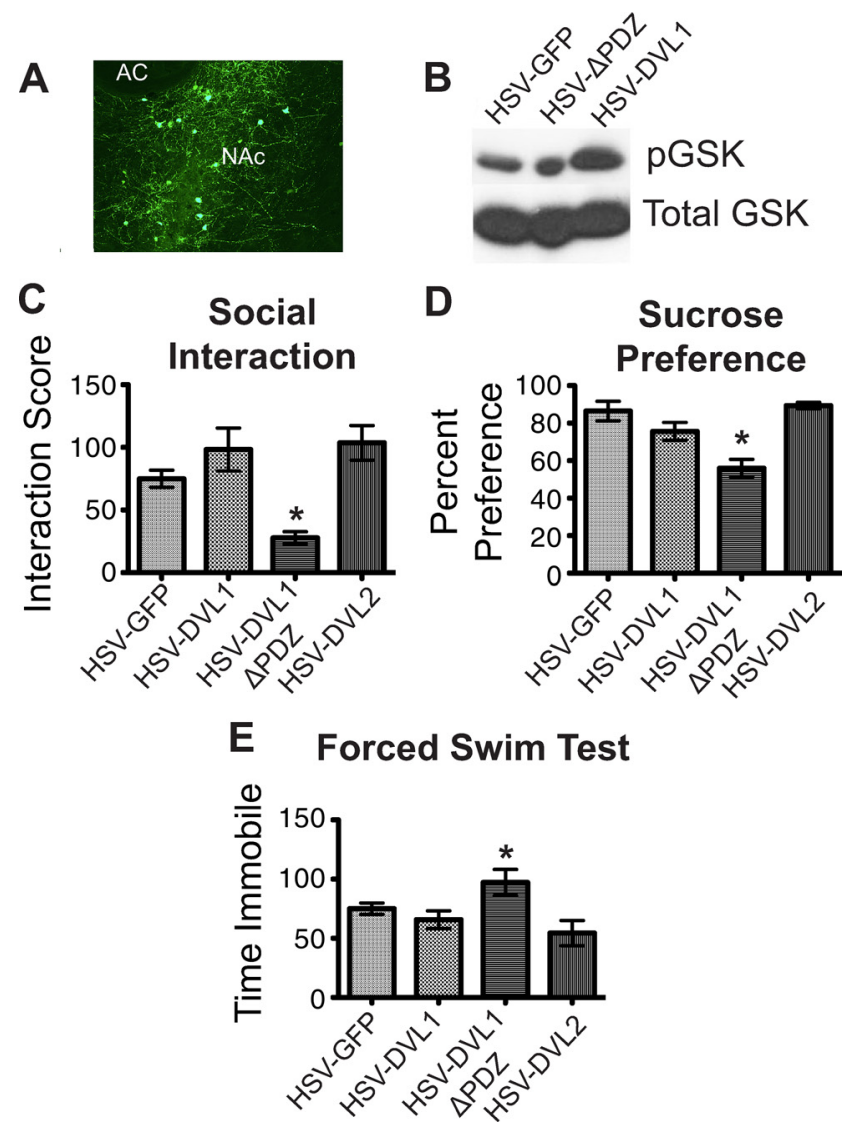

Figure 3. Downregulation of DVL activity in the NAc promotes depression-like behavior. $\boldsymbol{A}$, Representative example of HSV-mediated gene transfer in the mouse NAC. AC, Anterior commissure. $\boldsymbol{B}$, Western blot demonstrating the in vivo effectiveness of HSV-DVL1- $\triangle$ PDZ and HSVDVL1 as evidenced, respectively, by decreased and increased phospho (p)-GSK3 $\beta$ (GSK) levels in NAc. The blot is representative of several replicates. C, Animals receiving HSV-DVL1- $\triangle$ PDZ into the NAc showed decreased social interaction after submaximal defeat stress compared with mice receiving HSV-DVL1 or HSV-GFP ( $n=8-10$ per group). $\boldsymbol{D}, \boldsymbol{E}, \mathrm{HSV}-\mathrm{DVL}-\Delta \mathrm{PDZ}$ likewise decreased sucrose preference $(\boldsymbol{D}, n=8-10$ per group) and increased total time of immobility in the forced swim test $(\boldsymbol{E}, n=8-10$ per group), compared with HSV-GFP, with HSV-DVL exerting no effect. Error bars are SEM $\left({ }^{*} p<0.05\right)$.

HSV vector system infects neurons only, with approximately a third of neurons in the injection area affected, while producing no greater cytotoxic effects than seen with vehicle injections alone, and has been used with great success in numerous in vivo studies (Carlezon et al., 1998; Barrot et al., 2002; Neve et al., 2005; Krishnan et al., 2007, 2008; Covington et al., 2010). To validate the new DVL constructs, we overexpressed them in mouse NAc and demonstrated that HSV-DVL1 increases phospho-GSK3 $\beta$, while HSV-DVL1- $\triangle$ PDZ decreases phospho-GSK3 $\beta$, without affecting total levels of the protein, as would be expected (Fig. $3 B$ ).

We first tested whether overexpression of wild-type or mutant DVL altered the susceptibility of mice to social defeat stress by using a submaximal period of stress, which does not induce social avoidance in normal animals (Krishnan et al., 2007). Animals that received intra-NAc injections of HSV-GFP as a control showed levels of social interaction (Fig. 3C) roughly comparable to nondefeated mice, although somewhat lower due to interexperiment variability and the effect of intracranial surgery (Fig. $2 B)$. In contrast, animals that received intra-NAc injections of HSV-DVL1- $\triangle$ PZD developed significant social avoidance (Fig. $3 C$ ), whereas overexpression of wild-type HSV-DVL1 or HSVDVL2 had no significant effect (one-way ANOVA: $F_{(3,26)}=$ 
9.415, $p=0.0002$; there was no difference between the HSVDVL1 and HSV-DVL2 overexpression groups and control as shown by Tukey's post hoc test).

We next examined the effect of wild-type or mutant DVL in the sucrose preference test, where a reduction in sucrose consumption is used as a measure of anhedonia, indicative of a depression-like state (Moreau et al., 1992; Willner, 1997). Animals were presented with two bottles ( $1 \%$ sucrose and water) for the $4 \mathrm{~d}$ following HSV surgery and the percentage of sucrose consumed was measured. Consistent with the social avoidance measure after social defeat, we found that intra-NAc overexpression of HSV-DVL1- $\triangle \mathrm{PZD}$ significantly reduced sucrose consumption, compared with HSV-GFP-injected control mice, with no effect seen on overexpression of wild-type DVL1 or DVL2 (one-way ANOVA: $F_{(3,30)}=12.45, p<0.0001$; the lack of significance between DVL1 or DVL2 overexpression and the control group was shown by Tukey's post hoc test) (Fig. 3D).

The forced swim test is widely used as an acute screen for antidepressant-like activity (Porsolt et al., 1977), with acute exposure to an antidepressant reducing the amount of time a mouse is immobile when placed in a water bath. Mice that received intra-NAc injections of HSV-DVL1- $\triangle \mathrm{PZD}$, compared with HSV-GFP control mice, displayed the opposite effect, an increase in immobility (one-way ANOVA: $F_{(3,24)}=3.887, p=0.0214$ ) (Fig. 3E). Overexpression of wild-type DVL1 or DVL2 had no effect (Tukey's post hoc test). Together, these findings show that, in three distinct behavioral assays, downregulation of DVL function in NAc, which mimics DVL downregulation in susceptible mice after chronic social defeat stress, increases depression-like behavior.

The data reported above demonstrated that HSV-DVL1 or HSV-DVL2 infused into the NAc before a submaximal defeat paradigm had no effect on susceptibility. To further explore the possible effects of wild-type DVLs in social defeat, we used a more aggressive social defeat paradigm, which is known to induce a full extent of social avoidance in normal mice but over an accelerated time frame that is consistent with the limited duration of HSV expression (Krishnan et al., 2007). We found that neither HSVDVL1 nor HSV-DVL2 reduced the degree of social avoidance exhibited by HSV-GFP control animals subjected to this accelerated social defeat paradigm (data not shown). None of the viral vectors used affected social interaction times in nondefeated control animals (data not shown). These data indicate that, while reducing DVL function with DVL1- $\triangle \mathrm{PDZ}$ is sufficient to promote susceptibility, increasing DVL function with wild-type DVL1 or DVL2 is not sufficient to induce resilience.

\section{Pharmacological blockade of the DVL pathway promotes depression-like behavior}

To complement viral-mediated manipulation of DVL activity in the NAc, we studied whether pharmacological blockade of DVL signaling also results in a depression-like phenotype. We used sulindac, a commercially available compound, which has been shown, among other actions, to block canonical WNT signaling by disrupting the PDZ domain of DVL (Lee et al., 2009). Sulindac was infused bilaterally into the NAc by use of osmotic minipumps to deliver the drug at a constant rate over a 2 week period. We confirmed that sulindac infusions, compared with vehicle infusions, caused the expected biochemical effect-inhibition of the DVL pathway - as evidenced by a significant reduction in levels of phospho-GSK3 $\beta$ (one-way ANOVA: $F_{(3,29)}=4.375, p=$ 0.0117 ) (Fig. 4A), with no effect on the total amount of the protein or on levels of several control proteins (e.g., GAPDH,
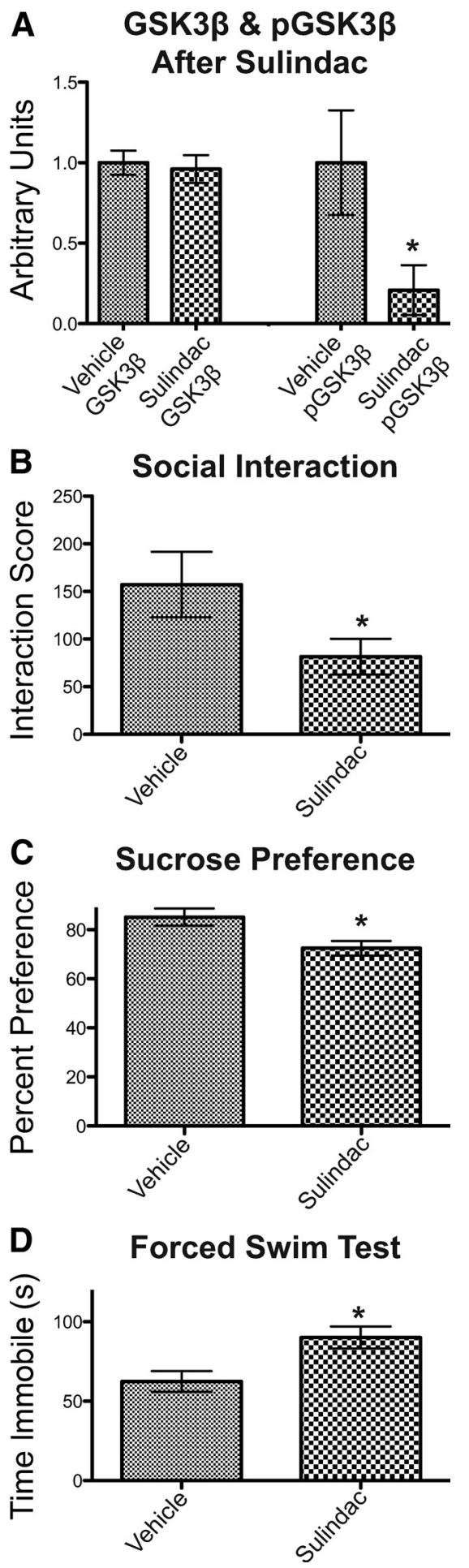

Figure 4. Pharmacological inhibition of DVL activity in the NAc promotes depression-like behavior. $A$, Intra-NAc infusion of sulindac significantly decreases levels of phospho-GSK3 $\beta$ (pGSK3 $\beta$ ), while total levels of GSK3 $\beta$ remain unchanged ( $n=8-10$ per group). $\boldsymbol{B}-\boldsymbol{D}$, IntraNAc administration of sulindac renders mice more susceptible to submaximal chronic social defeat stress ( $\boldsymbol{B}, n=6-8$ per group), as well as decreases sucrose preference $(\boldsymbol{C}, n=8-9$ per group), and increases immobility in the forced swim test ( $\boldsymbol{D}, n=8-9$ per group). Error bars are SEM $\left({ }^{*} p<0.05\right)$.

$\beta$-actin). Sulindac- and vehicle-infused mice were then analyzed in the same behavioral battery that was used in our HSV study above. Intra-NAc administration of sulindac, like intra-NAc overexpression of DVL1- $\triangle \mathrm{PDZ}$, rendered mice more susceptible 
to submaximal chronic social defeat stress (Student's $t$ test, $p=$ 0.037, $\mathrm{df}=14$ ) (Fig. 4 B), decreased sucrose preference (Student's $t$ test, $p=0.01, \mathrm{~d}=15)$ (Fig. 4C), and increased immobility in the forced swim test (Student's $t$ test $p=0.01, \mathrm{df}=15$ ) (Fig. $4 D$ ), all of which are prodepression-like effects. In contrast, sulindac had no effect on general levels of arousal based on normal levels and patterns of locomotor activity in an open field (data not shown).

\section{GSK3 $\beta$ overexpression recapitulates a depression-like phenotype}

As GSK3 $\beta$ is the primary mediator of DVL signaling in the canonical pathway (Gao and Chen, 2010), we were interested in assessing the behavioral consequences of viral manipulation of GSK $3 \beta$ in the NAc. Indeed, as stated earlier, levels of phosphoGSK3 $\beta$ are downregulated in the NAc of susceptible mice after chronic social defeat stress, as would be expected from a decrease in DVL function. Since a decrease in phospho-GSK3 $\beta$ reflects an increase in its catalytic activity, our hypothesis was that overexpression of wild-type GSK $3 \beta$, like overexpression of DVL1$\triangle \mathrm{PDZ}$, would promote depression-like behavior.

C57 mice received bilateral intra-NAc injections of HSVGSK $3 \beta$ or HSV-GFP as a control and were subjected to a submaximal course of chronic social defeat stress. As seen with DVL1- $\triangle$ PDZ, animals that overexpressed GSK $3 \beta$ in the NAc displayed greater susceptibility to defeat stress, as indicated by the development of social avoidance, which was not seen in GFPexpressing control mice: GFP animals showed a social interaction score of $80 \pm 7$ versus $52 \pm 2$ in GSK $3 \beta$ animals (Student's $t$ test, $p=0.023, \mathrm{df}=17)$. Similarly, mice overexpressing GSK3 $\beta$ in the NAc displayed a reduction in sucrose preference (one-way ANOVA: $F_{(2,21)}=11.19, p=0.0005$ ) (Fig. $5 A$ ) as well as an increase in immobility in the forced swim test (one-way ANOVA: $F_{(2,14)}=4.107, p=0.04$ ) (Fig. $5 B$ ). These findings support the view that the effect of DVL downregulation on depression-like behavior is mediated via DVL's canonical signaling pathway. In both paradigms, Tukey post hoc tests demonstrated no difference between the HSV-GFP-treated animals and those treated with HSV-dnGSK3 $\beta$ (the dominant-negative mutant of the enzyme).

We also considered the converse experiment whereby expression of dnGSK $3 \beta$ might decrease depression-like behavior. Mice received bilateral intra-NAc injections of either HSV-dnGSK3 $\beta$ or HSV-GFP and were then subjected to the accelerated paradigm of defeat stress described above, which induces maximal social avoidance in normal animals over the limited period of HSV expression (Krishnan et al., 2007). We found that overexpression of $\operatorname{dnGSK} 3 \beta$ in the NAc is sufficient to prevent the social avoidance induced by subsequent exposure to social defeat stress, as seen in GFP-expressing mice (Fig. 5C). Next, we were interested in examining whether overexpression of $\operatorname{dnGSK} 3 \beta$ could also reverse the deleterious effects of prior social defeat stress. To test this possibility, mice were subjected to the accelerated social defeat paradigm after which they received intra-NAc injections of HSV-dnGSK $3 \beta$ or HSV-GFP. Surprisingly, dnGSK $3 \beta$ overexpression in the NAc, poststress, did not reverse the social avoidance induced by social defeat stress (one-way ANOVA: $F_{(2,15)}=16.06, p=$ 0.0002; Tukey post hoc testing demonstrated no difference between HSV-GFP and HSV-dnGSK3 $\beta$ in the postdefeat treated animals) (Fig. $5 C$ ). These findings suggest that DVL-GSK $3 \beta$ pathway manipulation must be in place during the chronic stress to abrogate stressinduced behavioral abnormalities.

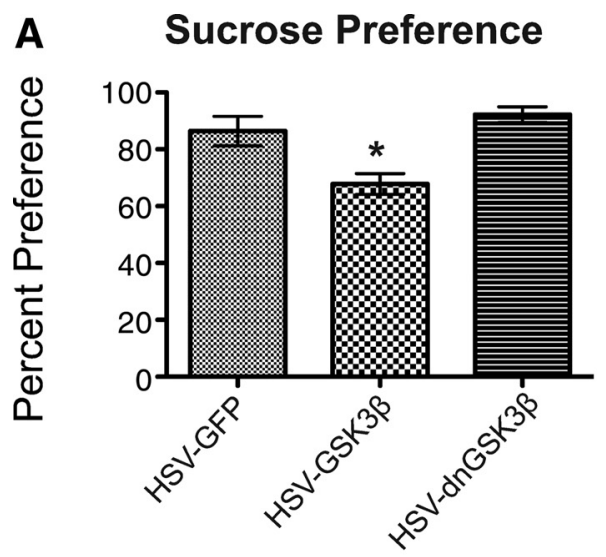

\section{B $\quad$ Forced Swim Test}

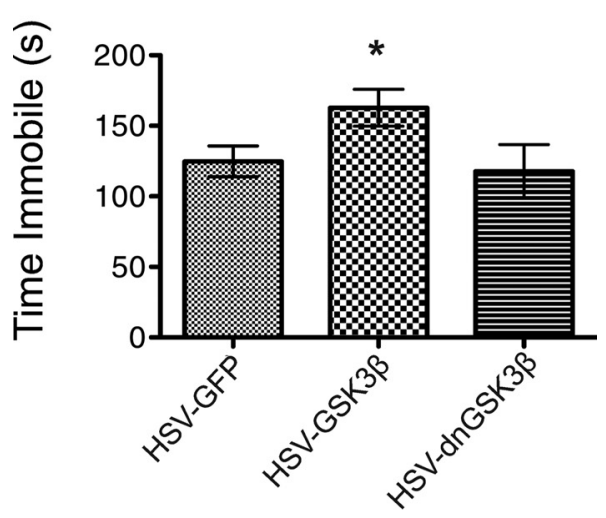

C Social Interaction

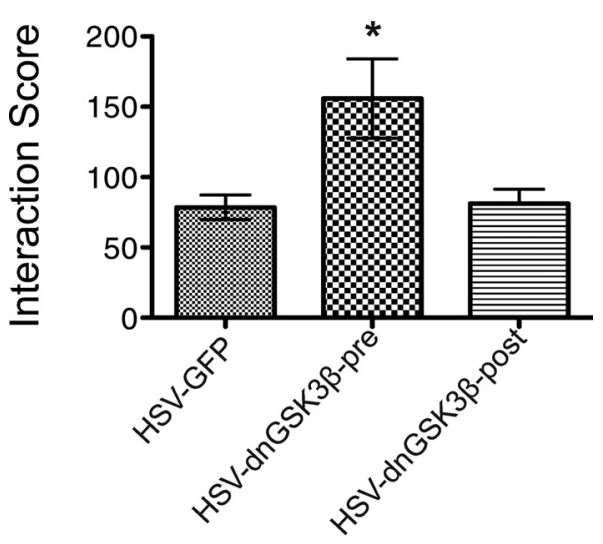

Figure 5. Behavioral effects of GSK3 $\beta$ manipulation in the mouse NAC. $A, B$, Similar prodepression-like effects of HSV-GSK3 $\beta$ were seen in the sucrose preference test $(A, n=6-8$ per group) and the forced swim test ( $\boldsymbol{B}, n=5-7$ per group). In both assays, there was no difference between the control virus (GFP) and dnGSK3 $\beta$. C, dnGSK3 $\beta$ attenuates avoidance in the social interaction test induced by the accelerated paradigm of chronic social defeat stress when expressed before, but not after, the stress ( $n=4-5$ per group). Error bars are SEM $\left({ }^{*} p<0.05\right)$.

\section{Discussion}

The current study demonstrates the usefulness of genome-wide chromatin analyses to identify novel targets in animal models of psychiatric disease. While the success of this approach has previously been shown in addiction models (Renthal et al., 2009; Maze et al., 2011), this is one of the first studies to do so in an animal model of depression. Based on the molecular pathway analysis of 
mRNA expression levels and of repressive histone methylation in the NAc in response to chronic social defeat stress (Wilkinson et al., 2009), we identified DVL2 as being significantly downregulated in susceptible mice, but not in resilient mice. We go on to establish the regulation of several proteins in the WNT-DVLGSK $3 \beta$ signaling cascade in this brain region of susceptible mice, and demonstrate directly that such altered signaling contributes to a depression-like phenotype in the social defeat paradigm and other behavioral assays.

We first show that there is widespread downregulation at the mRNA and protein level in the NAc of several genes in the WNTDVL-GSK3 $\beta$ pathway in response to chronic social defeat stress. While our previous work led us first to test DVL2 (Wilkinson et al., 2009), we found not only that the other known isoforms of DVL (DVL1 and DVL3) were downregulated by defeat stress, but also that several upstream and downstream members of the pathway (certain WNT family members, Frizzled receptors, Axin) were downregulated and that these adaptations are associated, as would be expected, with reduced levels of phospho-GSK3 $\beta$. Moreover, we demonstrated that these changes in DVLs were also found in the NAc of depressed humans examined postmortem, which provides important validation of our data in mice.

We next used viral-mediated gene transfer to directly downregulate activity of DVL proteins locally within the NAc of adult mice. We accomplished this by using a dominant-negative mutant of DVL, DVL1- $\triangle$ PDZ, which has been shown to block signaling by all DVL isoforms in several experimental systems and tissues (Krylova et al., 2000; Uematsu et al., 2003; Grandy et al., 2009; Gao and Chen, 2010). This mutation blocks DVL signaling since the PDZ domain is required for DVL polymerization, Axin binding, and downstream phosphorylation and inhibition of GSK $3 \beta$. We show that intra-NAc injection of HSV vectors expressing DVL1- $\triangle \mathrm{PDZ}$ is sufficient to increase susceptibility to a submaximal protocol of social defeat stress and to decrease sucrose preference test and increase immobility in the forced swim test, both prodepression-like effects. Similar findings were obtained when we pharmacologically inhibited the PDZ domain of DVL with local infusion of sulindac into the NAc. While sulindac is not selective for this action (it is best characterized as a cyclooxygenase inhibitor), it has been shown to decrease DVL signaling in the canonical pathway in peripheral tissues (Han et al., 2008), and we validated similar actions in the NAc upon direct infusion of the drug into this brain region. As found with DVL1$\triangle \mathrm{PDZ}$ overexpression, sulindac infusion increased susceptibility to chronic social defeat stress and induced prodepression-like effects in the sucrose preference and forced swim tests. It would be important to follow up these findings with more selective pharmacological inhibitors of DVL activity once such inhibitors become generally available.

Given the dramatic regulation of GSK3 $\beta$, the downstream effector of canonical DVL signaling, in the social defeat paradigm, we directly examined the influence of this enzyme in depression-like behavior. The dephosphorylated form of GSK3 $\beta$ is catalytically active and capable of phosphorylating $\beta$-catenin and several other downstream substrates. DVL inhibits GSK3 $\beta$ by disrupting the complex it normally forms with other proteinsincluding Axin, adenomatosis polyposis coli, and $\beta$-catenin-and thereby enabling its phosphorylation (at Ser9) by AKT (also known as protein kinase $\mathrm{B}$ ). Thus, downregulation of DVL, and of phosphoGSK $3 \beta$, in the NAc of susceptible mice would be expected to increase GSK3 $\beta$ catalytic activity and would support the hypothesis that GSK $3 \beta$ overexpression, like DVL inhibition, in this brain region would induce a prodepression-like effect. Indeed, we demonstrate here that overexpression of wild-type GSK $3 \beta$ induces a depressionlike phenotype including increased susceptibility to chronic social defeat stress, decreased sucrose preference, and increased immobility in the forced swim test. Conversely, we show that overexpression of a dominant-negative form of GSK $3 \beta$ in the NAc, before the social defeat paradigm, enhances resilience to subsequent stress.

Interestingly, we found that overexpression of $\operatorname{dnGSK} 3 \beta$ in the NAc did not reverse the deleterious effects of prior exposure to stress, suggesting that manipulation of GSK $3 \beta$ signaling may be most effective in prophylaxis to stress as opposed to being used to treat existing depression. Findings with wild-type DVL were even more striking: overexpression of DVL1 or DVL2 in this brain region neither prevented the deleterious effects of subsequent social defeat nor reversed the effects of prior defeat. These results may reflect the profound dysregulation of the WNT-DVLGSK3 $\beta$ signaling pathway both upstream and downstream of DVL (Table 3), thus rendering manipulation of DVL itself, as opposed to GSK $3 \beta$, unable to influence the effects of subsequent stress. These experiments illustrate the utility of our viral vectors and mouse models to explore which particular protein in the WNT-DVL-GSK3 $\beta$ pathway might be best suited for targeting with novel antidepressants.

Our work on DVL-GSK3 $\beta$ in the NAc in depression models is largely consistent with several recent findings performed in other brain regions. Okamoto et al. (2010) reported recently that chronic treatment with any of several antidepressants increases WNT signaling, including downstream regulation of DVL and GSK $3 \beta$, in hippocampus, effects opposite to those observed here in response to chronic stress. Also, consistent with our functional data showing a depression-like phenotype upon blockade of DVL signaling, Okamoto et al. (2010) found that viral-mediated overexpression of WNT2 in this brain region increased sucrose consumption and decreased the number of escape failures in the learned helplessness test, both antidepressant-like effects. In contrast, Dahlhoff et al. (2010) reported effects in the opposite direction: a single brief foot shock stress increased AKT signaling and decreased GSK $3 \beta$ activity in hippocampus and amygdala. While further research is needed to clarify these conflicting observations, the possible convergence of WNT-DVL-GSK3 $\beta$ regulation in hippocampus, with our findings in NAc, is striking, since many other proteins and biochemical pathways (e.g., BDNF-TrkB signaling, CREB, histone deacetylase-5, among others) have been found to play opposing roles in the NAc versus hippocampus in depression models (Nestler and Carlezon, 2006; Tsankova et al., 2006; Renthal et al., 2007). Such converging actions across multiple brain regions highlight the possibility that the WNT-DVLGSK3 $\beta$ cascade may be a particularly attractive one for the development of novel antistress treatments. This notion is supported by a recent study that demonstrated that a global knock-in (Ser9Ala) mutation of GSK3 $\beta$ to prevent its phosphorylation and inhibition increases susceptibility to depression-like behavior (Polter et al., 2010).

Indeed, a large amount of literature has long suggested the possible importance of GSK3 $\beta$ antagonism by lithium in the treatment of bipolar disorder (Davis et al., 1999; Kupka et al., 2003; Geddes et al., 2004), although there still is no direct evidence whether this or any of the many other known molecular actions of lithium mediates its antimanic properties. Moreover, there are unconfirmed reports of abnormal GSK $3 \beta$ signaling, as well as possible genetic variants, in bipolar and perhaps other mood disorders (Inkster et al., 2010; Pandey et al., 2010). The Inkster et al. (2010) study, which fits nicely with our data, also showed that polymorphisms in several WNT-DVL-GSK3 $\beta$ path- 
way genes, including DVL2 specifically, are correlated with depression and certain neuropathological findings. However, considerable work in the pharmaceutical industry to date suggests that GSK3 $\beta$ per se does not represent a viable drug target due to peripheral toxicity seen with systemic enzyme inhibition (Cohen and Goedert, 2004).

Accordingly, the present study, along with several others (Okamoto et al., 2010), are important because they provide a template that can be used to achieve net inhibition of GSK3 $\beta$ by targeting any of several upstream or downstream proteins that could potentially be better tolerated. While much future work is needed to study this possibility, results of the present study demonstrate a novel role for DVL and GSK3 $\beta$, acting in the NAc, in mediating the deleterious effects of chronic social defeat stress, and highlight the need for further research to delineate the exact downstream molecular pathways involved.

\section{References}

Barrot M, Olivier JD, Perrotti LI, DiLeone RJ, Benton O, Eisch AJ, Impey S, Storm DR, Neve RL, Zachariou V, Nestler EJ (2002) CREB activity in the nucleus accumbens shell controls gating of behavioral responses to emotional stimuli. Proc Natl Acad Sci U S A 99:11435-11440.

Berton O, McClung CA, Dileone RJ, Krishnan V, Renthal W, Russo SJ, Graham D, Tsankova NM, Bolanos CA, Rios M, Monteggia LM, Self DW, Nestler EJ (2006) Essential role of BDNF in the mesolimbic dopamine pathway in social defeat stress. Science 311:864-868.

Carlezon WA Jr, Thome J, Olson VG, Lane-Ladd SB, Brodkin ES, Hiroi N, Duman RS, Neve RL, Nestler EJ (1998) Regulation of cocaine reward by CREB. Science 282:2272-2275.

Clark MS, Sexton TJ, McClain M, Root D, Kohen R, Neumaier JF (2002) Overexpression of 5-HT1B receptor in dorsal raphe nucleus using Herpes simplex virus gene transfer increases anxiety behavior after inescapable stress. J Neurosci 22:4550-4562.

Cohen P, Goedert M (2004) GSK3 inhibitors: development and therapeutic potential. Nat Rev Drug Discov 3:479-487.

Covington HE 3rd, Maze I, LaPlant QC, Vialou VF, Ohnishi YN, Berton O, Fass DM, Renthal W, Rush AJ 3rd, Wu EY, Ghose S, Krishnan V, Russo SJ, Tamminga C, Haggarty SJ, Nestler EJ (2009) Antidepressant actions of histone deacetylase inhibitors. J Neurosci 29:11451-11460.

Covington HE 3rd, Lobo MK, Maze I, Vialou V, Hyman JM, Zaman S, LaPlant Q, Mouzon E, Ghose S, Tamminga CA, Neve RL, Deisseroth K, Nestler EJ (2010) Antidepressant effect of optogenetic stimulation of the medial prefrontal cortex. J Neurosci 30:16082-16090.

Dahlhoff M, Siegmund A, Golub Y, Wolf E, Holsboer F, Wotjak CT (2010) AKT/GSK-3beta/beta-catenin signalling within hippocampus and amygdala reflects genetically determined differences in posttraumatic stress disorder like symptoms. Neuroscience 169:1216-1226.

Davis JM, Janicak PG, Hogan DM (1999) Mood stabilizers in the prevention of recurrent affective disorders: a meta-analysis. Acta Psychiatr Scand 100:406-417.

Gao C, Chen YG (2010) Dishevelled: the hub of Wnt signaling. Cell Signal 22:717-727.

Geddes JR, Burgess S, Hawton K, Jamison K, Goodwin GM (2004) Longterm lithium therapy for bipolar disorder: systematic review and metaanalysis of randomized controlled trials. Am J Psychiatry 161:217-222.

Grandy D, Shan J, Zhang X, Rao S, Akunuru S, Li H, Zhang Y, Alpatov I, Zhang XA, Lang RA, Shi DL, Zheng JJ (2009) Discovery and characterization of a small molecule inhibitor of the PDZ domain of dishevelled. J Biol Chem 284:16256-16263.

Han A, Song Z, Tong C, Hu D, Bi X, Augenlicht LH, Yang W (2008) Sulindac suppresses beta-catenin expression in human cancer cells. Eur J Pharmacol 583:26-31.

Inkster B, Nichols TE, Saemann PG, Auer DP, Holsboer F, Muglia P, Matthews PM (2010) Pathway-based approaches to imaging genetics association studies: Wnt signaling, GSK3beta substrates and major depression. Neuroimage 53:908-917.

Krishnan V, Nestler EJ (2008) The molecular neurobiology of depression. Nature 455:894-902.

Krishnan V, Han MH, Graham DL, Berton O, Renthal W, Russo SJ, Laplant Q, Graham A, Lutter M, Lagace DC, Ghose S, Reister R, Tannous P, Green
TA, Neve RL, Chakravarty S, Kumar A, Eisch AJ, Self DW, Lee FS, et al. (2007) Molecular adaptations underlying susceptibility and resistance to social defeat in brain reward regions. Cell 131:391-404.

Krishnan V, Han MH, Mazei-Robison M, Iñiguez SD, Ables JL, Vialou V, Berton O, Ghose S, Covington HE 3rd, Wiley MD, Henderson RP, Neve RL, Eisch AJ, Tamminga CA, Russo SJ, Bolaños CA, Nestler EJ (2008) AKT signaling within the ventral tegmental area regulates cellular and behavioral responses to stressful stimuli. Biol Psychiatry 64:691-700.

Krylova O, Messenger MJ, Salinas PC (2000) Dishevelled-1 regulates microtubule stability: a new function mediated by glycogen synthase kinase3beta. J Cell Biol 151:83-94.

Kupka RW, Luckenbaugh DA, Post RM, Leverich GS, Nolen WA (2003) Rapid and non-rapid cycling bipolar disorder: a meta-analysis of clinical studies. J Clin Psychiatry 64:1483-1494.

Lee HJ, Wang NX, Shi DL, Zheng JJ (2009) Sulindac inhibits canonical Wnt signaling by blocking the PDZ domain of the protein Dishevelled. Angew Chem Int Ed Engl 48:6448-6452.

Maze I, Feng J, Wilkinson MB, Sun H, Shen L, Nestler EJ (2011) Cocaine dynamically regulates heterochromatin and repetitive element unsilencing in nucleus accumbens. Proc Natl Acad Sci U S A 108:3035-3040.

Moreau JL, Jenck F, Martin JR, Mortas P, Haefely WE (1992) Antidepressant treatment prevents chronic unpredictable mild stress-induced anhedonia as assessed by ventral tegmentum self-stimulation behavior in rats. Eur Neuropsychopharmacol 2:43-49.

Nestler EJ, Carlezon WA Jr (2006) The mesolimbic dopamine reward circuit in depression. Biol Psychiatry 59:1151-1159.

Neve RL, Neve KA, Nestler EJ, Carlezon WA Jr (2005) Use of herpes virus amplicon vectors to study brain disorders. Biotechniques 39:381-391.

Okamoto H, Voleti B, Banasr M, Sarhan M, Duric V, Girgenti MJ, Dileone RJ, Newton SS, Duman RS (2010) Wnt2 expression and signaling is increased by different classes of antidepressant treatments. Biol Psychiatry 68:521-527.

Pandey GN, Ren X, Rizavi HS, Dwivedi Y (2010) Glycogen synthase kinase3 beta in the platelets of patients with mood disorders: effects of treatment. J Psychiatr Res 44:143-148.

Polter A, Beurel E, Yang S, Garner R, Song L, Miller CA, Sweatt JD, McMahon L, Bartolucci AA, Li X, Jope RS (2010) Deficiency in the inhibitory serine-phosphorylation of glycogen synthase kinase- 3 increases sensitivity to mood disturbances. Neuropsychopharmacology 35:1761-1774.

Porsolt RD, Le Pichon M, Jalfre M (1977) Depression: a new animal model sensitive to antidepressant treatments. Nature 266:730-732.

Renthal W, Maze I, Krishnan V, Covington HE 3rd, Xiao G, Kumar A, Russo SJ, Graham A, Tsankova N, Kerstetter KA, Kippin TE, Neve RL, Haggarty SJ, McKinsey TA, Bassel-Duby R, Olson EN, Nestler EJ (2007) Histone deacetylase 5 epigenetically controls behavioral adaptations to chronic emotional stimuli. Neuron 56:517-529.

Renthal W, Kumar A, Xiao G, Wilkinson M, Covington HE 3rd, Maze I, Sikder D, Robison AJ, LaPlant Q, Dietz DM, Russo SJ, Vialou V, Chakravarty S, Kodadek TJ, Stack A, Kabbaj M, Nestler EJ (2009) Genome-wide analysis of chromatin regulation by cocaine reveals a role for sirtuins. Neuron 62:335-348.

Tsankova NM, Berton O, Renthal W, Kumar A, Neve RL, Nestler EJ (2006) Sustained hippocampal chromatin regulation in a mouse model of depression and antidepressant action. Nat Neurosci 9:519-525.

Uematsu K, Kanazawa S, You L, He B, Xu Z, Li K, Peterlin BM, McCormick F, Jablons DM (2003) Wnt pathway activation in mesothelioma: evidence of Dishevelled overexpression and transcriptional activity of beta-catenin. Cancer Res 63:4547-4551.

Vialou V, Robison AJ, Laplant QC, Covington HE 3rd, Dietz DM, Ohnishi YN, Mouzon E, Rush AJ 3rd, Watts EL, Wallace DL, Iñiguez SD, Ohnishi YH, Steiner MA, Warren BL, Krishnan V, Bolaños CA, Neve RL, Ghose S, Berton $\mathrm{O}$, Tamminga CA, Nestler EJ (2010a) $\Delta$ FosB in brain reward circuits mediates resilience to stress and antidepressant responses. Nat Neurosci 13:745-752.

Vialou V, Maze I, Renthal W, LaPlant QC, Watts EL, Mouzon E, Ghose S, Tamminga CA, Nestler EJ (2010b) Serum response factor promotes resilience to chronic social stress through the induction of DeltaFosB. J Neurosci 30:14585-14592.

Wilkinson MB, Xiao G, Kumar A, LaPlant Q, Renthal W, Sikder D, Kodadek TJ, Nestler EJ (2009) Imipramine treatment and resiliency exhibit similar chromatin regulation in the mouse nucleus accumbens in depression models. J Neurosci 29:7820-7832.

Willner P (1997) Validity, reliability and utility of the chronic mild stress model of depression: a 10-year review and evaluation. Psychopharmacology (Berl) 134:319-329. 